\title{
Calphostin C, a remarkable multimodal photodynamic killer of neoplastic cells by selective nuclear lamin B1 destruction and apoptogenesis (Review)
}

\author{
ANNA CHIARINI ${ }^{1}$, JAMES F. WHITFIELD ${ }^{2}$, RAFFAELLA PACCHIANA ${ }^{1}$, \\ MADDALENA MARCONI ${ }^{1}$, UBALDO ARMATO ${ }^{1}$ and ILARIA DAL PRÀ ${ }^{1}$ \\ ${ }^{1}$ Department of Biomedical and Surgical Sciences, University of Verona Medical School, 8 Strada Le Grazie, \\ I-37134 Verona, Italy; ${ }^{2}$ Institute for Biological Sciences, NRC, 52 Montreal Road, Ottawa, Ontario, K1A-0N6 Canada
}

Received October 13, 2009; Accepted December 2, 2009

DOI: 10.3892/or_00000711

\begin{abstract}
Perylenequinones that generate reactive oxygen species (ROS) when illuminated with visible light have been recommended as photodynamic chemotherapeutic agents. One of these is calphostin $\mathrm{C}(\mathrm{CalC})$, the action of the photoactivated derivative of which, $\mathrm{CalC}^{\varphi \mathrm{E}}$, has been ascribed to its ability to selectively and irreversibly inhibit protein kinase $\mathrm{Cs}$ (PKCs). But recent results of experiments with neoplastic rat fibroblasts and human breast and uterine cervix cancer cells have revealed that the action of $\mathrm{CalC}^{\varphi \mathrm{E}}$ involves more than PKC inhibition. Besides suppressing PKC activity, $\mathrm{CalC}^{\varphi \mathrm{E}}$ rapidly causes endoplasmic reticulum (ER) stress in breast cancer cells and the selective complete oxidation and proteasomal destruction of the functionally essential nuclear envelope protein lamin B1, in human cervical carcinoma (HCC) cells and neoplastic rat fibroblasts. When these lamin B1-lacking cells are placed in the dark, cytoplasmic membrane-linked PKC activities suddenly rebound and apoptogenesis is initiated as indicated by the immediate release of cytochrome $c$ from mitochondria and later on the activation of caspases. Hence, $\mathrm{CalC}^{\varphi \mathrm{E}}$ is a photodynamic cytocidal agent attacking multiple targets in cancer cells and it would be worth determining, even for their best applicative
\end{abstract}

Correspondence to: Professor Ubaldo Armato, Department of Biomedical and Surgical Sciences, University of Verona Medical School, 8 Strada Le Grazie, I-37134 Verona, Italy

E-mail: ubaldo.armato@univr.it

Abbreviations: ANT, adenine nucleotide translocase; CalC, calphostin $\mathrm{C}$; $\mathrm{CalC}^{\varphi \mathrm{E}}$, photoactivated $\mathrm{CalC}$; $\mathrm{CPF}$, cytoplasmic particulate fraction; DAG, diacylglycerol; ER, endoplasmic reticulum; HCC, human cervical carcinoma; NL, nuclear lamina; NMF, nuclear membrane fraction; PKC, protein kinase C; ROS, reactive oxygen species; VDAC, voltage-dependent anion protein

Key words: apoptogenesis, human neoplastic cells, lamins, perylenequinones, polyomavirus-transformed rat fibroblasts, reactive oxygen species use, whether other perylenequinones also share the so far unexpectedly complex deadly properties of the $\mathrm{CalC}^{\varphi \mathrm{E}}$.

\section{Contents \\ 1. Introduction \\ 2. The light-induced events and their in-dark sequelae \\ 3. The drivers of $\mathrm{CalC}^{\varphi \mathrm{E}}$ cytocidal actions \\ 4. Conclusions}

\section{Introduction}

Perylenequinones are second-generation cell photosensitizers which are easily purified, highly soluble, amenable to sitedirected chemical modifications, quickly inactivated in the dark and rapidly cleared from tissues without a persisting tissue photosensitivity. Therefore, the perylenequinones have been recommended as photodynamic therapeutic agents (1-6).

Calphostin C (CalC; UCN-1028c) is one of these agents. It is a dark red to brown, highly lipophilic, protein kinase $\mathrm{C}$ (PKC)-inhibiting, perylenequinone metabolite from the fungus Cladosporium cladosporioides (Fig. 1A) (7-12). It has a high molecular extinction coefficient $(\varepsilon)$, a wide UVvisible absorption spectrum with peaks at 226 ( $\varepsilon$ 44,800), 269 ( $\varepsilon 29,550), 474$ ( $\varepsilon 23,550), 553.9$ ( $\varepsilon$ shoulder 11,900) and $582 \mathrm{~nm}(\varepsilon 12,000)$, and a high quantum yield of ${ }^{1} \mathrm{O}_{2}$ generation in aqueous solution $(5,12,13)$. According to these properties $\mathrm{CalC}$ absorbs enough near infrared light to be photoexcited to $\mathrm{CalC}^{\varphi \mathrm{E}}$ for use for photodynamic tumor therapy (5).

$\mathrm{CalC}^{\varphi \mathrm{E}}$ is best known and used for its photodependent strong ability to selectively inhibit most PKC isoforms (7-13). However, it has recently been shown that $\mathrm{CalC}^{\varphi \mathrm{E}}$ mounts a multi-target attack on ER (endoplasmic reticulum) glycoprotein transport resulting in ER vacuolation, ER stress, and a cluster of apoptogenic consequences [i.e., activation of cJun N-terminal kinase and protein kinase R-like ER kinase and upregulation of CCAAT/enhancer-binding protein homologous transcription factor (CHOP/GADD153)] independently of the inhibition of PKCs activity (4). But $\mathrm{CalC}^{\varphi \mathrm{E}}$ also rapidly and selectively attacks the neoplastic cell nucleus (14). 
A
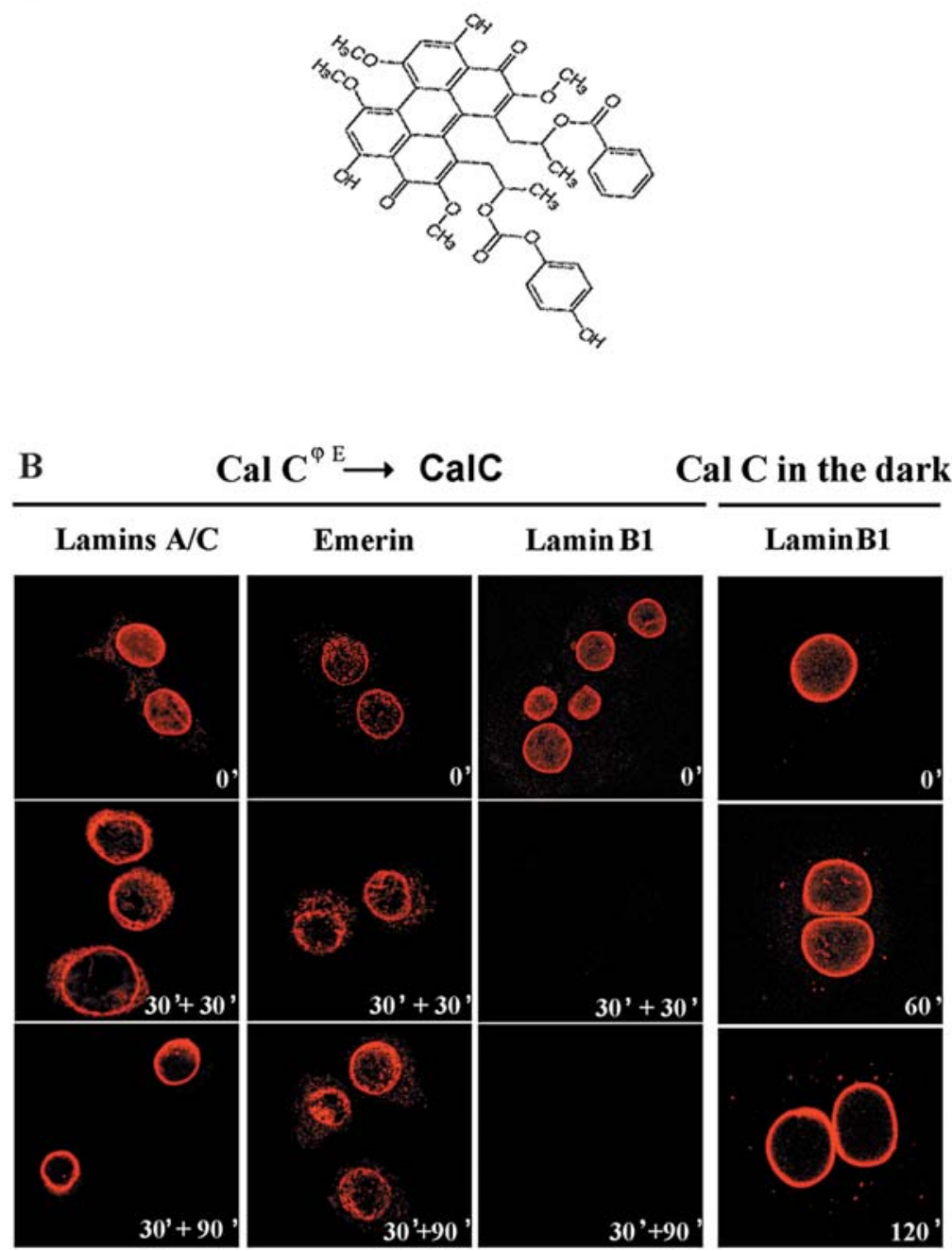

C Lamin B1 at NM fraction

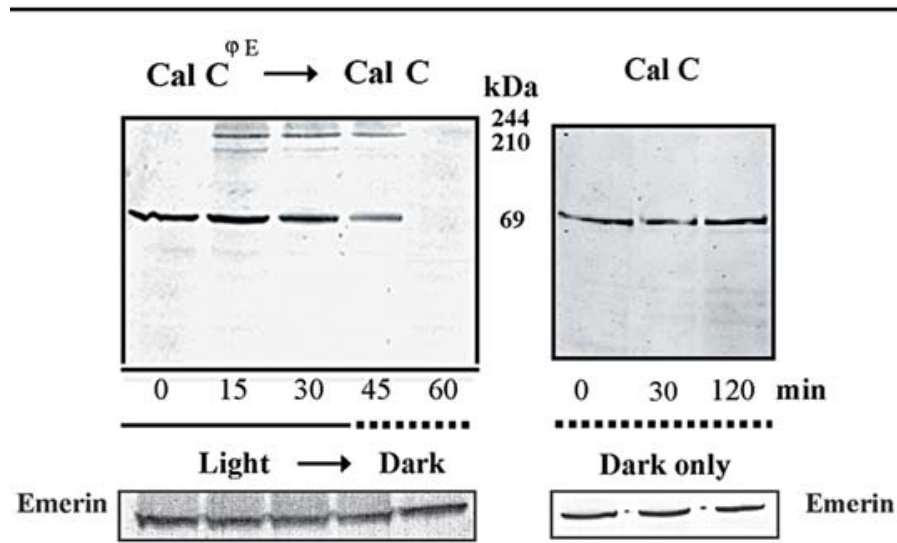

Figure 1. The photoactivation of $\mathrm{CalC}$ to $\mathrm{CalC}^{\varphi \mathrm{E}}$ sets off the rapid and complete destruction of the survival-essential lamin $\mathrm{B} 1$ at the nuclear envelope. (A) The chemical structure of CalC fully justifies its lipophilicity and ability to enter cells and to be enriched in the cytoplasmic membranes (ER and Golgi apparatus) and nuclear envelope. However, photoactivation is necessary for the biological effects of $\mathrm{CalC}^{\varphi \mathrm{E}}$, like destruction of lamin $\mathrm{B} 1$, inhibition of PKCs (IC50 $=50 \mathrm{nM}$ ), and apoptogenesis, to be made happen. If never exposed to light since the onset of the experiments, cell-permeating CalC is totally harmless (14). (B) Lamins $\mathrm{A} / \mathrm{C}$ and emerin were not changed by a 30-min exposure to $\mathrm{Cal} \mathrm{C}^{\textrm{E}}$ followed by a 30-90-min incubation in the dark still in the presence of the rapidly inactivated $\mathrm{CalC}$; by neat contrast, lamin B1 was totally destroyed within 60 min by the same treatment; strikingly, lamin B1 was not altered by a 60-120-min direct exposure to CalC but no light (14). (C) On the left, the time-related progressive disappearance of 69-kDa lamin B1 holoprotein and the transient appearance of 210- and 244-kDa predestruction bands recognized by the antilamin B1 antibody in the nuclear membrane fractions (NMFs) of cells exposed for $30 \mathrm{~min}$ to $\mathrm{CalC}^{\textrm{E}}$ and next incubated in the dark with no medium change; the 0-min band is from illuminated only control cells. Conversely, NMF-associated 69-kDa lamin B1 did not wane and no anti-lamin B1 antibody-recognized 210- and 244-kDa bands obtained in the NMFs when cells were exposed to $\mathrm{CalC}$ but no light. In either instance, equal sample loading was monitored by reprobing the immunoblots with the anti-emerin antibody. The immunofluorescent pictures (B) and the immunoblots (c) are typical of five distinct experiments (14). 
One of first, if not its first, critical target of $\mathrm{CalC}^{\varphi \mathrm{E}}$, is the nuclear lamina (NL). This is the fibrous network that lines the inner surface of the nuclear envelope (15-20). The lamina consists basically of 3-D bundles of lamins $\mathrm{A} / \mathrm{C}$ piled on top of a sheet of parallel filaments of isoprenylated lamin B filaments joined at $15-\mathrm{nm}$ intervals along their length by short (e.g., 5-nm), non-continuous cross-linkers (15-20). It anchors the nuclear pores plus various other structural and functional components to the nuclear envelope and its periphery (15-20). It controls the transcription of some genes by sequestering transcription factors such as c-Fos and B-catenin and preventing them from getting to their target genes (21). Isoprenylated lamin B 1 is permanently attached to the inner nuclear membrane orienting and stabilizing the nucleus in the cell by attaching it to the surrounding cytoskeleton and, for example, preventing the nucleus from spinning (22). Lamin B is the ancestral lamin that is expressed by all cells at all stages of development and, unlike the more mobile lamins $\mathrm{A} / \mathrm{C}$, it is essential for the structural integrity of the nuclear envelope and normal nuclear functioning. Therefore, knocking down lamin B1 and B2 proteins with RNAi apoptotically kills cells such as human cervical carcinoma (HCC) HeLa S6 cells and F5 and FR (wt648) rat fibroblasts, while knocking down lamins $\mathrm{A} / \mathrm{C}$ displaces the associated emerin, but does not kill the cells (23).

We have recently reported that loading polyomavirustransformed pyF111 rat fibroblasts as well as HCC C4-I cells, in an in vitro model for late-stage invasive human cervical cancer (24), with $\mathrm{CalC}$ and producing $\mathrm{CalC}^{\varphi \mathrm{E}}$ by illuminating them with visible light for $30 \mathrm{~min}$, rapidly and selectively destroys lamin B1 (14). Despite the layering of bundles of lamin A/C on the targeted lamin B1 (17), they are not destroyed (Fig. 1B and C), nor are the lamin A/C-associated emerin and the nucleoplasmic cyclin $\mathrm{E}$ affected (14). CalC ${ }^{\textrm{E}}$ is also apoptogenic $(14,25)$. Surprisingly, apoptogenesis does not start in neoplastic rat fibroblasts and HCC C4-I cells until lamin B1 has been mostly destroyed and $\mathrm{CalC}{ }^{\varphi \mathrm{E}}$ turned off by transferring the lamin B1-depleted cells in a dark incubator (14).

Here, we review the emerging case for a new multimodal model for CalC photodynamic action consisting not just of PKC inhibition and ER stress, but of light-induced selective lamin B1 destruction and PKC inhibition followed, after transfer to the dark, by rebounding cytoplasmic PKC- $\beta_{I}$ and PKC- $\delta$ activities and the onset of apoptogenesis.

\section{The light-induced events and their in-dark sequelae}

$\mathrm{CalC}^{\varphi \mathrm{E}}$ rapidly enters the cytoplasm of cells and, like hypericin (6), accumulates mainly in the Golgi apparatus and the ER membranes triggering ER stress $(4,5)$. It does not enter the nucleus, although because of its high lipophilicity it probably also collects in the ultimately ER-derived cell nuclear envelope (18). During the first $30 \mathrm{~min}$ of illumination, the nuclear envelope lamin $\mathrm{B} 1$ of the $\mathrm{CalC}^{\varphi \mathrm{E}}$-loaded transformed rat fibroblasts or HCC C4-I cells undergoes a brisk, progressive decline and totally disappears during the first 30 min of incubation in the dark (where $\mathrm{CalC}^{\varphi \mathrm{E}}$ is rapidly inactivated to CalC) (14) (Fig. 1B and C). While the lamin $\mathrm{B} 1$ is being destroyed, neither nuclear membrane lamins
$\mathrm{A} / \mathrm{C}$ nor the protein emerin, the lamin A-associated binder of transcription factors and part of the four-protein nuclear motor (containing nuclear actin, emerin, lamin A, and nuclear myosin) that moves chromosomes to reposition genes in response to various signals $(21,26)$, are affected $(14)$. The nucleoplasmic cyclin $\mathrm{E}$ is also untouched even though within the first $10 \mathrm{~min}$ of illumination the aqueous peroxides resulting from the cytoplasmic-located, short-range firing, ephemeral reactive oxygen species $\left(\mathrm{ROS},{ }^{1} \mathrm{O}_{2}\right.$ and $\mathrm{O}_{2^{-}}$) produced by the photoactivated $\mathrm{CalC}^{\varphi \mathrm{E}}$ significantly increase their levels in the nucleus (14).

During the 30 min of illumination and $\mathrm{CalC}^{\varphi \mathrm{E}}$ activity in the cytoplasm, cytoplasmic PKC- $\beta_{\mathrm{I}}$ and $\mathrm{PKC}-\delta$ activities rapidly drop close to zero, as expected (Fig. 2A and B) $(25,27)$. There are no indications of incipient apoptogenesis such as mitochondrial cytochrome $c$ release or caspase-3 activation (Fig. 2C and D) $(14,27)$. However, after the cells are transferred to the dark, while lamin B1 destruction continues to completion, the photo-suppressed cytoplasmic particulate fraction (CPF)-associated PKC- $\beta_{\mathrm{I}}$ and $\mathrm{PKC}-\delta$ activities rebound within minutes (Fig. $2 \mathrm{~A}$ and $\mathrm{B})(25,27)$.

The prompt in-dark resumption of $\mathrm{PKC}$ activities in the CPF is accompanied by the first sign of apoptogenesis (28), the rapid-onset release of mitochondrial cytochrome $c$ (Fig. 2C). This release then slows down between 60 and $120 \mathrm{~min}$ and finally levels off between 120 and $240 \mathrm{~min}$ (Fig. 2C). The cytochrome $c$ release is followed by a steadily increasing activity of executioner caspase-3 starting around 90 min (Fig. 2D) (25), and more than $90 \%$ of the cells are killed by $3.5 \mathrm{~h}$ after shutting off the light $(14,27)$.

Finally, it must be noted that none of these ultimately lethal events, the destruction of lamin B1 or apoptogenesis, are triggered by incubating the pyF111 cells with $75 \mathrm{nM}$ unexcited $\mathrm{CalC}$ in the dark. With $\mathrm{CalC}$, but without $\mathrm{CalC}^{\varphi \mathrm{E}}$, the cells keep their normal lamin B1-supported nuclear envelopes, continue proliferating normally and double their numbers by $24 \mathrm{~h}$ in the dark $(14,27)$.

\section{The drivers of $\mathrm{CalC}^{\varphi \mathrm{E}}$ cytocidal actions}

The early intranuclear accumulation of aqueous peroxides derived from ${ }^{1} \mathrm{O}_{2}$ and $\mathrm{O}_{2}$ - produced by cytoplasm-located, lipophilic $\mathrm{CalC}^{\varphi \mathrm{E}}$ might be the cause of the so far inexplicably selective oxidation of the nuclear envelope of lamin B1, whereas the closely placed lamin A or lamin A-associated protein emerin or the intranuclear cyclin $\mathrm{E}$ are simultaneously spared (14). The oxidized lamin B1, like any other oxidized proteins (29), is in turn proteolyzed by a stimulated proteasomal peptidyl-glutaminase-like protease (14). The turned-on proteasome has an ongoing inertia. So, turning off the light and with it $\mathrm{CalC}^{\varphi \mathrm{E}}$ at 30 min does not stop oxidized lamin B1 proteolysis from going to completion in as many minutes (Fig. 1C) (14).

Different PKCs have also been shown to be involved in apoptogenesis as well as proliferation and other activities $(25,27,30,31)$. Therefore, as expected, when $\mathrm{CalC}^{\varphi \mathrm{E}}$ is driving oxidized lamin B1 destruction, it silences PKC- $\beta_{\mathrm{I}}$ and PKC- $\delta$ and any indication of impending apoptogenesis (Fig. 2A-D). Thus, for example, as the activities of the PKC- $\delta$ and PKC- $\beta_{I}$ 
A

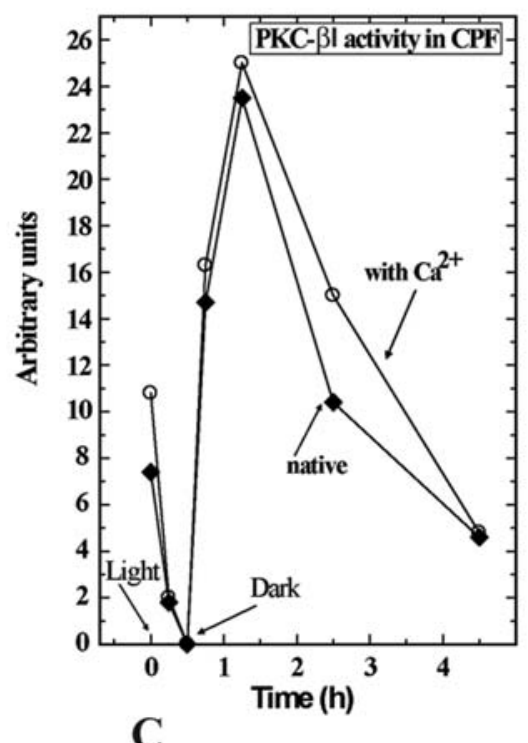

C

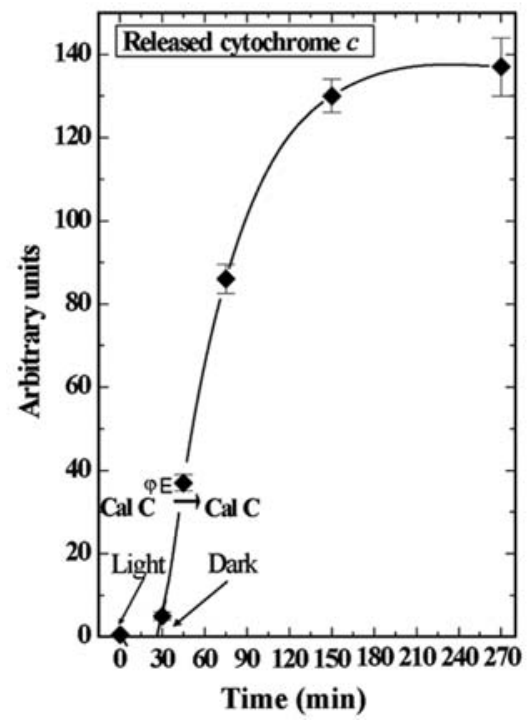

B
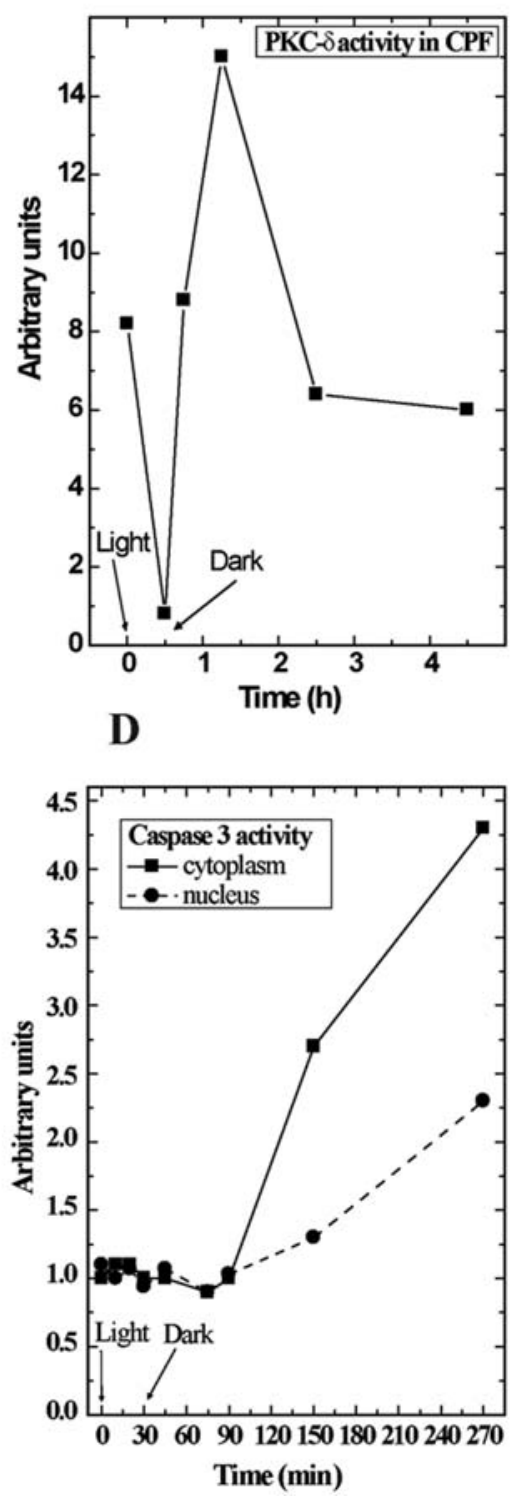

Figure 2. The very fast rebounding of the activities of the CPF-linked PKC- $\beta_{I}$ and PKC- $\delta$ from the $C a C^{\varphi E}-i n d u c e d$ transient inhibition after transfer to the dark concurs with the brisk onset of apoptogenic events. (A and $\mathrm{B}$ ) Exposure to $\mathrm{CalC}^{\varphi \mathrm{E}}$ elicited within 30 min the virtually total inhibition of immunoprecipitable $\mathrm{PKC}-\beta_{\mathrm{I}^{-}}$and $\mathrm{PKC}-\delta$-specific activities linked to the $\mathrm{CPF}$. However, soon after $\mathrm{CalC}^{\varphi \mathrm{E}}$ was inactivated to CalC by transfer to a dark incubator, the CPF-linked immunoprecipitable PKC- $\beta_{\Gamma^{-}}$and PKC- $\delta$-specific activities promptly rebounded. SEMs, not shown, were within $\pm 12 \%$ the corresponding mean values $(25,27$ and previously unpublished). (C) A massive release of cytochrome $c$ from mitochondria into the cytosol occurred soon after $\mathrm{CalC}^{\varphi \mathrm{E}}$ was inactivated to CalC by transfer to a dark incubator, and was concomitant with the rebounding CPF-linked PKC- $\beta_{\Gamma^{-}}$and PKC- $\delta$ activities, but preceded the activation of executioner caspase-3. Every point on the curves is the mean value \pm SEM of three distinct experiments, each carried out in triplicate (25). (D) The activation of apoptotic caspase-3 in $\mathrm{CalC}^{\varphi \mathrm{E}}$-exposed pyF111 cells occurred well downstream of the total waning of NMF-associated lamin B1. The levels of caspase-3 activity in both the nuclear and cytoplasmic fraction remained unchanged for up to 90 min (30-min exposure to CalC $\mathrm{C}^{\varphi \mathrm{E}}+$ 60-min incubation with dark-inactivated CalC) prior to surging during the following $180 \mathrm{~min}$ of staying in the dark. Points on the curves are means from 3-5 independent experiments. SEMs (data not shown) were within $\pm 11 \%$ of the mean values (14).

holoenzymes bound to the CPF drop virtually to zero by 30 min. During the first $30 \mathrm{~min}$ after $\mathrm{CalC}^{\varphi \mathrm{E}}$ is turned off in the dark, the PKC- $\beta_{I}$ and PKC- $\delta$ activities in the CPF, to which they translocate, surge very sharply upwards (Fig. 2A and B) (27). This sudden and large rebound from PKC- $\beta_{I}$ and $\mathrm{PKC}-\delta$ inactivity is unexpected because of the alleged irreversibility of PKC inhibition in $\mathrm{C} 6$ glioma cells by $\mathrm{CalC}^{\varphi \mathrm{E}}$ (7). Gopalakrishna et al (8) reported that washing $\mathrm{CalC}$ out of the glioma cells did not immediately restore PKC activity. More precisely, re-establishment of PKC activity took $12 \mathrm{~h}$ instead of the few minutes it took in the transformed rat fibroblasts $(24,27)$ and thus required the reloading of the glioma cells with newly made PKCs. This might mean either that the photoinactivation of $\mathrm{PKCs}$ by $\mathrm{CalC}^{\varphi \mathrm{E}}$ in the pyF111 cells is in fact reversible or that these cells have a store of inactive non-membrane-associated PKCs that are 
thus inaccessible to lipophilic $\mathrm{CalC}^{\varphi \mathrm{E}}$ and its ROS photoproducts, but can be recruited to cytoplasmic membranes and there swiftly activated when $\mathrm{CalC}^{\varphi \mathrm{E}}$ disappears.

\section{Conclusions}

It is generally believed that $\mathrm{CalC}^{\varphi \mathrm{E}}$ owes its ability to act as a potential photodynamic chemotherapeutic agent by selectively and irreversibly preventing growth factor- or tumor promoter-stimulated PKCs from driving the proliferation of neoplastic cells $(32,33)$. Since it is highly lipophilic, CalC probably collects in cell membranes where the photoexcited CalC ${ }^{\varphi \mathrm{E}}$ can fire ${ }^{1} \mathrm{O}_{2}$ and $\mathrm{O}_{2}$ - onto PKCs that have been recruited there. The bombardment of a membrane-associated PKC with ${ }^{1} \mathrm{O}_{2}$ and $\mathrm{O}_{2}$ from $\mathrm{CalC}{ }^{\varphi \mathrm{E}}$ prevents diacylglycerol (DAG) released from the membrane's phospholipids by a receptoractivated phospholipase $\mathrm{C}$ or a tumor-promoting phorbol ester from binding to the irreversibly altered DAG/phorbol ester kinase binding site in its $\mathrm{C} 1$ domain, which normally would activate the enzyme by causing its pseudosubstrate domain to lift away from, and stop blocking, the catalytic domain (8,33-36).

But such a PKC-blocking-only mechanism appears be only half or even less of the story for at least the neoplastic rat fibroblasts and $\mathrm{HCC}$ C4-I cells. Instead, $\mathrm{CalC}^{\varphi \mathrm{E}}$ is a multiple attacking cytocidal agent with at least two of its targets being equally lethal. When photoexcited in the cytoplasm, the CalC ${ }^{\varphi \mathrm{E}}$-generated ${ }^{1} \mathrm{O}_{2}$ and $\mathrm{O}_{2}$ and their hydroperoxide derivatives selectively oxidize and thereby target the nuclear membrane indispensible lamin B1 for seemingly total destruction (14). As expected, at the same time $\mathrm{CalC}^{\varphi \mathrm{E}}$ inhibits cytoplasmic PKC- $\beta_{\mathrm{I}}$ and $\mathrm{PKC}-\delta$. But contrary to expectations $(8,35)$, turning off the light promptly unleashes $\mathrm{PKC}-\beta_{\mathrm{I}}$ and $\mathrm{PKC}-\delta$ activities in the $\mathrm{CPF}$, which coincides with the release of mitochondrial cytochrome $c(27)$ that starts the apoptogenic caspase cascade (28).

A possible $\mathrm{CalC}^{\varphi \mathrm{E}}$-inhibitable mechanism by which the surging PKCs might trigger cytochrome $c$ release and apoptogenesis is suggested by Chan et al (36). They have shown that treating rat rostral ventrolateral medulla cells with Escherichia coli lipopolysaccharide causes the translocation of cytosolic PKC (including PKC- $\beta_{\mathrm{I}}$ and $\mathrm{PKC}-\delta$ )/Bax complexes to cell membranes, where Bax is released and next translocated to mitochondria. There, Bax forms complexes with ANT (adenine nucleotide translocase) or VDAC (voltage-dependent anion protein) that cause the opening of the mitochondrial permeability transition pore, the release of the apoptogenic cytochrome $c$ into the cytoplasm, and the activation of the apoptogenic caspases.

Clearly other perylenequinones should now be studied to see if this remarkable multimodal lethal capability of $\mathrm{CalC}^{\varphi \mathrm{E}}$ for neoplastic cells is shared by all the members of this family of photodynamic therapeutic agents.

\section{Acknowledgements}

This work was supported in part by the Italian MIUR (Ministero dell'Istruzione, dell'Università e della Ricerca, ex-60\% allotment funds) and by MIUR-PRIN (Cofin) funds.

\section{References}

1. Beck TP, Kirsh EJ, Chmura SJ, Kovar DA, Chung T, Rinker-Schaeffer CW and Stadler WM: In vitro evaluation of calphostin $\mathrm{C}$ as a novel agent for photodynamic therapy of bladder cancer. Urology 54: 573-577, 1999.

2. Diwu Z and Lown JW: Photosensitization with anticancer agents. 15. Perylenequinoid pigments as potential photodynamic therapeutic agents: formation of semiquinone radicals and reactive oxygen species on illumination. J Photochem Photobiol B 18: 131-143, 1993.

3. Gomer CJ: Preclinical examination of first and second generation photosensitizers used in photodynamic therapy, Photochem Photobiol 54: 1093-1107, 1991.

4. Kaul A and Maltese WA: Killing of cancer cells by the photoactivatable protein kinase $\mathrm{C}$ inhibitor, calphostin $\mathrm{C}$, involves induction of endoplasmic reticulum stress. Neoplasia 11: 823-834, 2009.

5. Olivo $\mathrm{M}$ and Ali-Seyed $\mathrm{M}$ : Apoptosis signaling mechanisms in human cancer cells induced by calphostin-PDT. Int J Oncol 30: 537-548, 2007

6. Sanovic R, Krammer B, Grumboeck S and Verwanger T: Timeresolved gene expression profiling of human squamous cell carcinoma cells during the apoptosis process induced by photodynamic treatment with hypericin. Int J Oncol 35: 921-939, 2009.

7. Bruns RF, Miller FD, Merriman RL, Howbert JJ, Heath WF, Kobayashi E, Takahashi I, Tamaoki T and Nakano H: Inhibition of protein kinase $\mathrm{C}$ by calphostin $\mathrm{C}$ is light-dependent. Biochem Biophys Res Commun 176: 288-293, 1991.

8. Gopalakrishna R, Chen $\mathrm{ZH}$ and Gundimeda U: Irreversible oxidative inactivation of protein kinase $\mathrm{C}$ by photosensitive inhibitor calphostin C. FEBS Lett 314: 149-154, 1992.

9. Kobayashi E, Ando K, Nakano H and Tamaoki T: UCN$1028 \mathrm{~A}$, a novel and specific inhibitor of protein kinase $\mathrm{C}$ from Cladosporium. J Antibiotics (Tokyo) 42: 153-155, 1989.

10. Kobayashi E, Nakano H, Morimoto $\mathrm{M}$ and Tamaoki T: Calphostin C (UCN-1028c), a novel microbial compound, is a highly potent and specific inhibitor of protein kinase $\mathrm{C}$. Biochem Biophys Res Commun 159: 548-553, 1989.

11. Pollack IF and Kawecki S: The effect of calphostin C, a potent photodependent protein kinase $\mathrm{C}$ inhibitor, on the proliferation of glioma cells in vitro. J Neurooncol 31: 255-266, 1997.

12. Takao I, Kobayashi E, Yoshida M and Sano H: Calphostins, novel and specific inhibitors of protein kinase C. B. Chemical structures. J Antibiotics (Tokyo) 42: 1475-1481, 1989.

13. Gueded RC and Eriksson LA: Photophysics, photochemistry, and reactivity: molecular aspects of perylenequinone reactions. Photochem Photobiol Sci 6: 1089-1096, 2007.

14. Chiarini A, Whitfield JF, Pacchiana R, Armato U and Dal Pra I: Photoexcited calphostin C selectively destroys nuclear lamin B1 in neoplastic human and rat cells. A novel mechanism of action of a photodynamic tumor therapy agent. Biochim Biophys Acta 1783: 1642-1653, 2008.

15. Bridger JM, Foeger N, Kill LR and Hermann H: The nuclear lamina. Both a structural framework and a platform for genome organization. FEBS J 274: 1354-1361, 2007.

16. Dechat T, Pfleghaar K, Sengupta K, Shimi T, Schumaker DK, Solimando L and Goldman RD: Nuclear lamins: major factors in the structural organization and function of the nucleus and chromatin. Genes Dev 22: 832-853, 2008.

17. Goldberg MW, Fiserova J, Huttenlauch I and Stick R: A new model for nuclear lamina organization. Biochem Soc Trans 36: 1339-1343, 2008.

18. Larijani B and Poccia DL: Nuclear envelope formation: mind the gaps. Annu Rev Biophys 38: 107-124, 2009.

19. Goldman RD, Gruenbaum Y, Moir RD, Schumaker DK and Spann TP: Nuclear lamins: building blocks of nuclear architecture. Genes Dev 16: 533-547, 2002.

20. Gruenbaum Y, Margalit A, Goldman RD, Schumaker DK and Wilson KL: The nuclear lamin comes of age. Nat Rev Mol Cell Biol 6: 21-31, 2005.

21. Heessen S and Fornerod M: The inner nuclear envelope as a transcription factor resting place. EMBO Rep 8: 914-919, 2007 . 
22. Ji JY, Lee RT, Vergnes L, Fong LG, Stewart CL, Reue K, Young SG, Zhang Q, Shanahan CM and Lammerding J: Cell nuclei spin in the absence of lamin B1. J Biol Chem 282: 20015-20026, 2007.

23. Harborth J, Elbashir SM, Bechert K, Tuschl T and Weber K: Identification of essential genes in cultured mammalian cells using small interfering RNAs. J Cell Sci 114: 4557-4565, 2001.

24. Carlson MW, Iyer VR and Marcotte EM: Quantitative gene expression assessment identifies appropriate cell line models for individual cervical cancer pathways. BMC Genomics 8: 117, 2007.

25. Dal Pra I, Whitfield JF, Chiarini A and Armato U: Increased activity of the protein kinase- $\delta$ holoenzyme in the cytoplasmic particulate fraction precedes activation of caspases in the polyomavirus-transformed pyF111 rat fibroblasts exposed to calphostin C or topoisomerase II inhibitors. Exp Cell Res 255: 171-183, 2000.

26. Mehta IS, Elcock LS, Amira M, Kill IR and Bridger JM: Nuclear motors and nuclear structures containing A-type lamins and emerin: is there a functional link? Biochem Soc Trans 36: 1384-1388, 2008.

27. Chiarini A, Whitfield JF, Armato U and Dal Pra I: VP-16 (etoposide) and calphostin $\mathrm{C}$ trigger different nuclear but akin cytoplasmic patters of changes in the distribution and activity of protein kinase $C-\beta_{I}$ in polyomavirus-transformed pyF111 rat fibroblasts. Int J Mol Med 17: 111-120, 2006.

28. Yan N and Shi Y: The mechanisms of apoptosis through structural biology. Annu Rev Cell Dev Biol 21: 35-56, 2005.
29. Jung T and Grune T: The proteasome and its role in the degradation of oxidized proteins. IUBMB Life 60: 743-752, 2008.

30. Chiarini A, Whitfield JF, Armato U and Dal Pra I: Protein kinase $\mathrm{C}-\mathrm{\beta}_{\mathrm{II}}$ is an apoptotic lamin kinase in polyomavirustransformed etoposide-treated pyF111 rat fibroblasts. J Biol Chem 277: 18827-18839, 2002.

31. Reyland ME: Protein kinase C- $\delta$ and apoptosis. Biochem Soc Trans 35 (Pt. 5): 1001-1004, 2007.

32. Da Rocha AB, Mans DRA, Regner A and Schwartsmann G: Targeting protein kinase C: new therapeutic opportunities against high-grade malignant gliomas? Oncologist 7: 17-33, 2002.

33. Whitfield JF and Chakravarthy B: Calcium: The Grand-Master Cell Signaller. NRC Research Press, Ottawa, 2001.

34. Newton AC: Protein kinase C: structure, function and regulation. J Biol Chem 270: 28495-28498, 1995.

35. Rotenberg SA, Huang MH, Zhu J, Su L and Riedel H: Deletion analysis of protein kinase $\mathrm{C}$ inactivation by calphostin C. Mol Carcinogenesis 12: 42-49, 1995.

36. Chan JY, Chang AY, Wang LL, Ou CC and Chan SH: Protein kinase C-dependent mitochondrial translocation of proapoptotic protein Bax on activation of inducible nitric-oxide synthase in rostral ventrolateral medulla mediates cardiovascular depression during experimental endotoxemia. Mol Pharmacol 71: 1129-1139, 2007. 